\title{
Stochastic collocation analysis of the transient current induced along the wire buried in a lossy medium
}

\author{
S. Šesnić ${ }^{1}$, S. Lalléchère ${ }^{2}$, D. Poljak ${ }^{1}$, P. Bonnet $^{2} \&$ K. E. K. Drissi ${ }^{2}$ \\ ${ }^{1}$ FESB, University of Split, Croatia \\ ${ }^{2}$ Institut Pascal, Université Blaise Pascal, France
}

\begin{abstract}
The paper deals with the stochastic collocation analysis of a time domain response of a straight thin wire scatterer buried in a lossy half-space. The wire is excited by a plane wave transmitted through the air-ground interface. Transient current induced at the centre of the wire, governed by corresponding Pocklington integro-differential equation is determined. This configuration, as is the case with many electromagnetic compatibility (EMC) issues, suffers from uncertainties in various parameters, such as ground properties, wire dimensions, position, etc. The obtained results yield additional statistical information thus enabling more accurate and efficient analysis of buried wire configurations.

Keywords: buried thin wire, analytical solution, Lossy half-space, stochastic collocation technique.
\end{abstract}

\section{Introduction}

There has been a continuous interest in the analysis of ground penetrating radar (GPR) systems and related applications in civil engineering [1]. Consequently, a deeper insight of scattering phenomena occurring in a lossy half-space, as well as the development of uncertainty analysis is required [2,3], This paper deals with the analysis of random variations regarding geometries, environment and/or materials of interest that may lead to substantial misunderstanding or errors in the analysis of buried objects. Due to uncertain variations of parameters of interest, (e.g. inability to obtain precise input parameters or environmental drifts caused by ground humidity) some techniques for an efficient integration of stochastic modelling have been developed [4]. This paper underlines the 
advantages of stochastic collocation (SC) method and aims to demonstrate the ability of a precise and analytical deterministic method to compute the current induced on a straight buried wire, combined with an efficient and accurate stochastic method (SC) to integrate uncertainties surrounding parameters accuracy.

\section{Antenna theory formulation and analytical solution}

A horizontal thin wire scatterer of length $L$ and radius $a$, is buried in a lossy medium at depth $d$. Properties of the medium are given with $\varepsilon$ and $\sigma$, i.e. electrical permittivity and conductivity, respectively. The wire is illuminated by a transmitted part of a transient electromagnetic (EM) wave of normal incidence (as shown in Fig. 1).

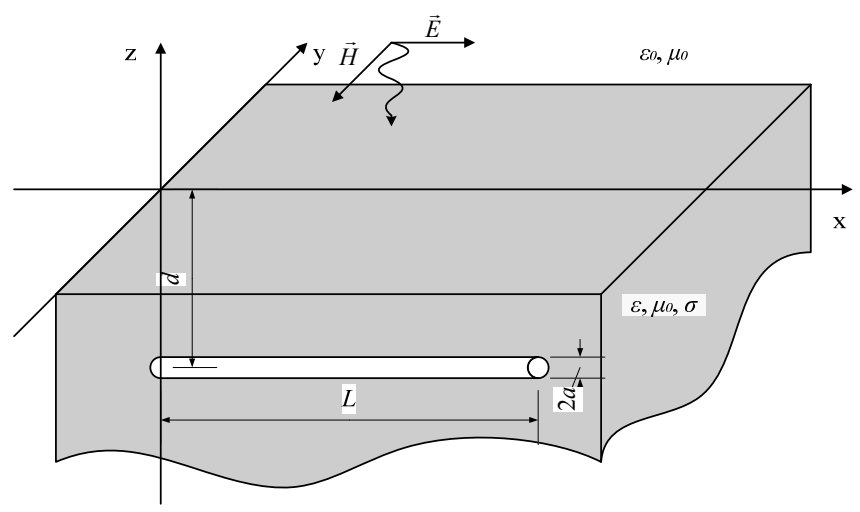

Figure 1: $\quad$ A horizontal thin wire buried in a lossy medium.

Time domain formulation for the transient analysis of horizontal straight wire buried in a lossy medium is based on the space-time Pocklington integrodifferential equation given with [5]

$$
\begin{aligned}
& \left(\mu \varepsilon \frac{\partial}{\partial t}+\mu \sigma\right) E_{x}^{t r}(t)=-\left(\frac{\partial^{2}}{\partial x^{2}}-\mu \sigma \frac{\partial}{\partial t}-\mu \varepsilon \frac{\partial^{2}}{\partial t^{2}}\right)
\end{aligned}
$$

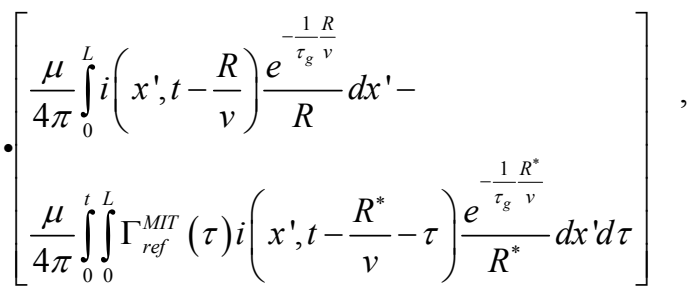


where $i\left(x^{\prime}, t-\frac{R}{v}\right)$ is the unknown space-time dependent current, $E_{x}^{t r}$ is the tangential transmitted field and $\Gamma_{r e f}^{M I T}$ is the corresponding reflection coefficient arising from the Modified Image Theory (MIT) [6].

The distance from the source point in the wire axis to the observation point located on the wire surface is

$$
R=\sqrt{\left(x-x^{\prime}\right)^{2}+a^{2}},
$$

while, the distance from the source point on the image wire to the observation point on the original wire, according to the image theory is

$$
R^{*}=\sqrt{\left(x-x^{\prime}\right)^{2}+4 d^{2}} .
$$

Time constant and propagation velocity in the lossy medium are given by

$$
\begin{aligned}
& \tau_{g}=\frac{2 \varepsilon}{\sigma}, \\
& v=\frac{1}{\sqrt{\mu \varepsilon}} .
\end{aligned}
$$

The influence of the earth-air interface is taken into account via the reflection coefficient arising from the MIT and is given with [5]

$$
\Gamma_{r e f}^{M I T}(t)=-\left[\frac{\tau_{1}}{\tau_{2}} \delta(t)+\frac{1}{\tau_{2}}\left(1-\frac{\tau_{1}}{\tau_{2}}\right) e^{-\frac{t}{\tau_{2}}}\right],
$$

where the corresponding time constants are

$$
\begin{gathered}
\tau_{1}=\frac{\varepsilon_{0}\left(\varepsilon_{r}-1\right)}{\sigma}, \\
\tau_{2}=\frac{\varepsilon_{0}\left(\varepsilon_{r}+1\right)}{\sigma} .
\end{gathered}
$$

Note that the reflection coefficient (5) represents rather simple characterization of the earth-air interface, taking into account only medium properties. An accuracy of (5) has been discussed in [5] and [7].

Undertaking the analytical solution procedure documented in [7], the expression for the time dependent induced current for the case of impulse excitation can be written as follows 


$$
i(x, t)=\frac{4 \pi}{\mu}\left\{\begin{array}{l}
R\left(s_{\Psi}\right)\left[1-\frac{\cosh \left(\gamma_{\Psi}\left(\frac{L}{2}-x\right)\right)}{\cosh \left(\gamma_{\Psi} \frac{L}{2}\right)}\right] e^{\left(t+\frac{a}{v}\right) s_{\Psi}} \\
-\frac{\pi}{\mu \varepsilon L^{2}} \sum_{n=1}^{\infty} \frac{2 n-1}{ \pm \sqrt{b^{2}-4 c_{n}} s_{1,2 n} \Psi\left(s_{1,2 n}\right)} \sin \frac{(2 n-1) \pi x}{L} e^{\left(t+\frac{a}{v}\right) s_{1,2 n}}
\end{array}\right\},
$$

where coefficients $R\left(s_{\Psi}\right)$ and $s_{\Psi}$ represent physical properties of the system, taking into account the dimensions of the wire and the distance from the interface:

$$
\begin{aligned}
& R\left(s_{\Psi}\right)=\frac{1}{2 \ln \frac{L}{2 d} \frac{s_{\Psi}}{s_{\Psi} \tau_{2}+1}\left(\tau_{1}-\tau_{2} \frac{s_{\Psi} \tau_{1}+1}{s_{\Psi} \tau_{2}+1}\right)}, \\
& s_{\Psi}=-\frac{\ln \frac{L}{a}+\ln \frac{L}{2 d}}{\tau_{1} \ln \frac{L}{a}+\tau_{2} \ln \frac{L}{2 d}} .
\end{aligned}
$$

Furthermore, other coefficients in (7) correspond to the properties of the medium and are given as follows:

$$
\begin{gathered}
\gamma_{\Psi}=\sqrt{\mu \varepsilon\left(s_{\Psi}^{2}+b s_{\Psi}\right)} \\
s_{1,2 n}=\frac{1}{2}\left(-b \pm \sqrt{b^{2}-4 c_{n}}\right) \\
b=\frac{\sigma}{\varepsilon} \\
c_{n}=\frac{(2 n-1)^{2} \pi^{2}}{\mu \varepsilon L^{2}}, n=1,2,3, \ldots .
\end{gathered}
$$

Expression (7) represents the impulse response. Consequently, the response to an arbitrary excitation requires convolution. In this paper, the normal incidence is considered, i.e. the plane wave in the form of the double exponential function is assumed

$$
E_{x}(t)=E_{0}\left(e^{-\alpha t}-e^{-\beta t}\right)
$$

The transmitted electric field exciting the buried wire in the Laplace domain is given by [8]

$$
E_{x}^{t r}(s)=\Gamma_{t r}(s) E_{x}(s) e^{-\gamma d}
$$

where $\Gamma_{t r}(s)$ represents Fresnel transmission coefficient defined by relation [7] 


$$
\Gamma_{t r}(s)=\frac{2 \sqrt{s \varepsilon_{0}}}{\sqrt{s \varepsilon+\sigma}+\sqrt{s \varepsilon_{0}}} .
$$

As the convolution, i.e. the time domain counterpart of (11), would be too complex to be calculated analytically, the numerical convolution is carried out, as it is presented in [7].

\section{Statistical and stochastic strategies}

Among the huge diversity of statistical approaches available in the literature, the purpose of this paper is to focus on spectral stochastic techniques [9]. Relying on the physical problem under consideration, the statistics of the current $I$ induced in the centre of the buried wire $\left(\left[I_{u}\right.\right.$, where $u$ represents a given order of statistical moment) is expanded over an adapted function basis $\left(\Phi^{u}(\mathbf{X})\right.$ depending on statistical order $u$ ) as follows:

$$
[I]_{u}(\mathbf{X}) \approx \sum_{v_{1}=0}^{n_{1}} \ldots \sum_{v_{N}=0}^{n_{N}} \eta_{w}^{v_{1} \ldots v_{N}} \Phi^{u}(\mathbf{X})
$$

where $\eta_{w}^{v_{1} \ldots v_{N}}$ stand for the weights of the expansion for random vector $\mathbf{X}$ of size $N\left(\mathbf{X}=\left(X_{1}, X_{2}, \ldots, X_{N}\right)^{T}\right.$ thus modelling the $N$ random components or parameters of the problem). Relation (13) involves the necessity for $n_{w}+1 \mathrm{SC}$ points (SC $n_{w}$ order expansion) to compute random component $X_{w}$ in a straightforward manner. Some more details related to this approach are available in [10].

\subsection{Multiple independent random variables principle}

The fundamentals of SC technique [10] applied to the buried wire configuration taking into account three Random Variables (3-RVs), are outlined. The principle through which one operates with a random output $I$ (current) depending on random parameters (ground conductivity $\sigma$ in $\mathrm{mS} / \mathrm{m}$, wire length $L$ and depth $d$ in $\mathrm{m}$ ) is illustrated. As depicted in (13), the SC technique is compatible with higher $\mathrm{RV}$ dimensions. The problem of interest requires one to model 3-RVs $\hat{u}_{1}, \hat{u}_{2}$ and $\hat{u}_{3}$ (randomly modelled physical parameters) respectively $X_{1}, X_{2}$ and $X_{3}$. The random variations related to independent $X_{1}, X_{2}$ and $X_{3}$ may be defined from the initial values $X_{1}^{0}, X_{2}^{0}$ and $X_{3}^{0}$. Applying the same strategy as documented in [10], function $(r, s, t) \rightarrow\left(I\left(X_{1}^{0}, X_{2}^{0}, X_{3}^{0} ; r, s, t\right)\right)$ is projected on a Lagrangian basis

$$
I\left(X_{1}^{0}, X_{2}^{0}, X_{3}^{0} ; r, s, t\right) \approx \sum_{i=0}^{n_{1}} \sum_{i=0}^{n_{2}} \sum_{i=0}^{n_{3}} I_{i j k}\left(X_{1}^{0}, X_{2}^{0}, X_{3}^{0}\right) L_{i}(r) L_{j}(s) L_{k}(t)
$$

where $I_{i j k}\left(X_{1}^{0}, X_{2}^{0}, X_{3}^{0}\right)=I\left(X_{1}^{0}, X_{2}^{0}, X_{3}^{0} ; r_{i}, s_{j}, t_{k}\right), r_{i}, s_{j}$ and $t_{k}$ are the SC points required in a corresponding random direction (i.e. accordingly to $X_{1}, X_{2}$ and $X_{3}$ parameters). 


\subsection{Computation of SC statistical moments: advantages and drawbacks}

As previously stated, the computation of output $I$ statistics from (14) through a tensor product in each direction (i.e., for each RV) is rather simple. The SC technique gives the collocation sets of weighted points necessary to compute the needed statistics (e.g. mean and standard deviation). However, the technique also requires a particular attention regarding the cost/benefit ratio when increasing $\mathbf{X}$ dimensions (tensor product of RV). Some methods for the improvement of stochastic techniques have been presented in [11] and [12]. The part to follow proposes an alternative strategy to iteratively (i.e. increasing one RV at a time) and completely construct a random model.

\section{Numerical results from an iterative construction of the random model}

This section deals with some numerical results and statistics considering the current at the centre of the wire buried in a lossy ground. The entire stochastic modelling is based upon realistic values of:

- $\quad$ soil conductivity $\sigma . \sigma=\sigma^{0}+\hat{u}_{1}^{0}$ with $\sigma^{0}=X_{1}^{0}=5 \mathrm{mS} / \mathrm{m}$ and $\hat{u}_{1}^{0}$ a zeromean RV with a uniform distribution from 1 to $9 \mathrm{mS} / \mathrm{m}$;

- length $L$ of wire: $L=L^{0}+\hat{u}_{2}^{0}$ with $L^{0}=X_{2}^{0}=10 \mathrm{~m}$ and $\hat{u}_{2}^{0}$ a zero-mean RV with a uniform distribution from 9.5 to $10.5 \mathrm{~m}$;

- $\quad$ burial depth $d$ : $d=d^{0}+\hat{u}_{3}^{0}$ with $d^{0}=X_{3}^{0}=4 \mathrm{~m}$ and $\hat{u}_{3}^{0}$ a zero-mean RV with a uniform distribution from 2.5 to $5.5 \mathrm{~m}$.

Without loss of generality, the problem can be addressed following different assumptions about the statistical distribution laws.

\subsection{Numerical results with one random variable}

In a first step, the mean value and variances including only one RV, soil conductivity, length of the wire or burying depth (RV1, RV2 and RV3, respectively) can be readily computed from (14). Figs 2, 3 and 4 show the deterministic and stochastic results obtained for the transient current at the centre of the buried wire. Although it is expected that soil conductivity is a crucial parameter, the results from Figs 2, 3 and 4 reinforce statistically this analysis. As shown in Figs 2, 3 and 4, the deterministic value of the mean transient current somewhat differs from the stochastic one. The average current $\langle I\rangle$ and its statistical dispersion $\langle I\rangle \pm s t d(I)$ are given in Figs 2, 3 and 4 and differences can be observed between their respective variances (i.e. standard deviations). In a first step, this may offer a quick glance on the sensitivity of the three parameters to uncertain variations. The SC convergence is obtained with a limited number of points in each case:

- 7 points are required including only soil conductivity (Fig. 2); 
- 3 points are necessary considering only the length of the wire (Fig. 3);

- 7 points may be sufficient to compute properly mean and variance of the burial depth (Fig. 4).

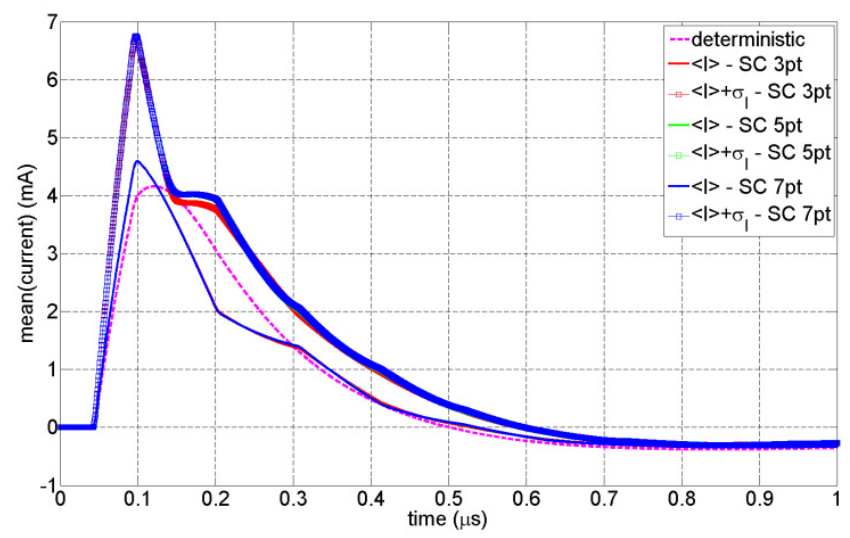

Figure 2: Current at the centre of the wire relying on RV1 $(\sigma)$.

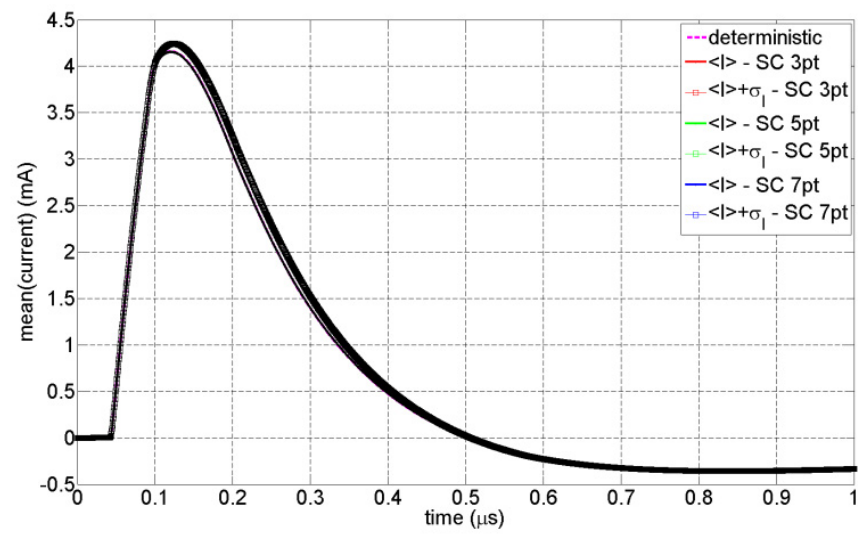

Figure 3: Current at the centre of the wire relying on RV2 $(L)$.

\subsubsection{Variance convergence in relation to system sensitivity}

This part focuses on the SC requirements for variance convergence to improve the knowledge of the model sensitivity regarding each parameter independently. However, the interactions between RVs are not taken into account, but a view of the SC convergence and an idea of the RV global sensitivity are given.

First, it is compulsory to ensure the number of SC points necessary to assess convergence by computing statistics of the current. Based upon criterion proposed in [10], the SC convergence from SC method with $s_{w}$ weighted points for RV number $w$ is given by 


$$
G_{w}\left([I]_{u}^{s_{w}}\right)=\left|\frac{[I]_{u}^{s_{w}}-[I]_{u}^{s_{w}+2}}{[I]_{u}^{s_{w}}}\right|
$$

where $[I]_{u}^{s_{w}}$ is the $u$-th order statistical moment computed from SC.

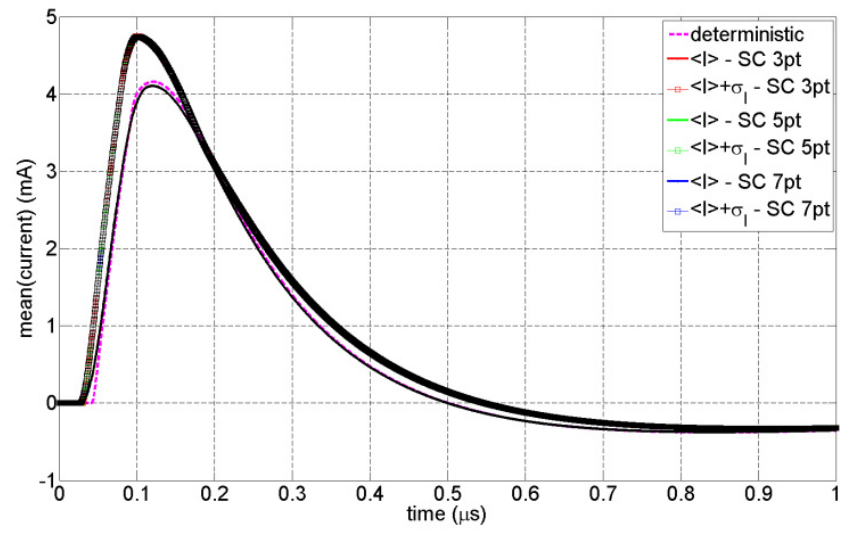

Figure 4: Current at the centre of the wire relying on RV3 (d).

Figure 5 shows the results obtained by applying relation (15) for RV1, RV2 and RV3. As expected, fewer points are expected to ensure high convergence rate for RV2 $(L)$ than for RV1 $(\sigma)$ and RV3 $(d)$.

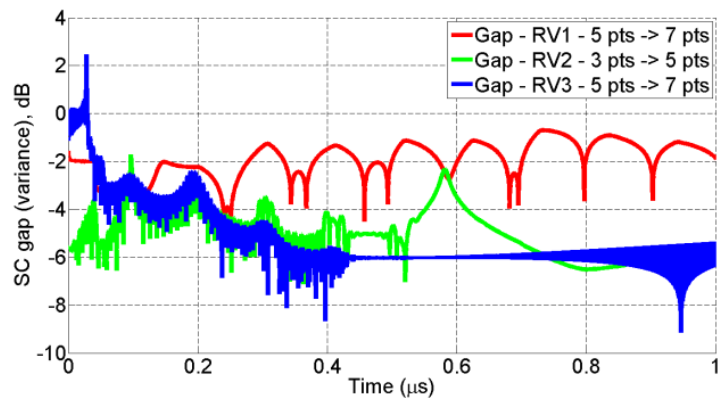

Figure 5: Relative gap (variance of the current) while increasing SC orders for 1-RV stochastic models.

Figure 6 emphasizes the importance of RV1 over other RVs: the relative dispersion from soil conductivity is larger than the one given by the length of the wire. An intermediate level is expected from RV2 (burial depth). Figure 6 gives a quick overview of the 1-RV model sensitivity throughout the simulation time. 


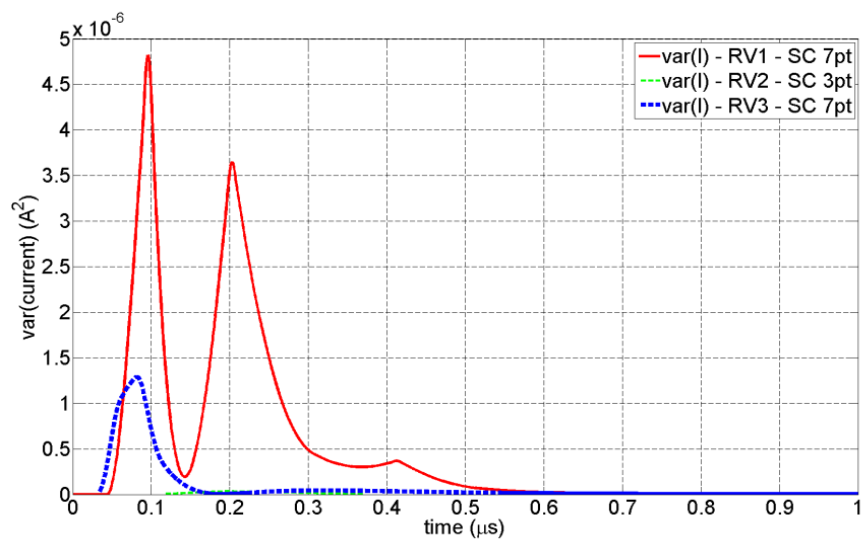

Figure 6: Variances of current computed from different stochastic modelling.

\subsection{Numerical results for entire random model}

This section deals with the numerical results obtained from the stochastic model including three selected random parameters.

\subsubsection{Fully tensorized modelling}

Figure 7 shows mean (+ one standard deviation) of the current at the centre of the wire under uncertain constraints fully tensorized (i.e. with $3 \mathrm{RVs}$ ). First, the similarity with results depicted in Fig. 2 is noteworthy (emphasizing the importance of RV1). The main difficulty relies on the number of samples needed to assess converged statistics: $3^{3}+5^{3}+7^{3}+9^{3}=1224$ points are required to ensure the asymmetrical convergence of 6-th order (i.e. 7 points for each RV).

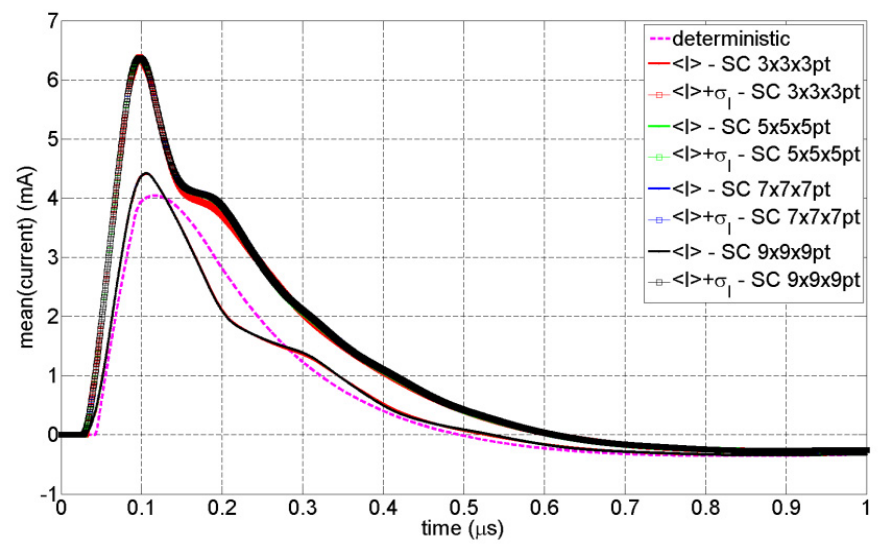

Figure 7: Currents at the centre of the wire ("full-tensor" model). 
The sensitivity analysis provides relevant information needed to decrease the total number of SC points required for each RV and optimise the "full tensor" random model to an "asymmetrical" one.

\subsubsection{3-RV full tensor optimization (asymmetrical SC)}

Figure 8 provides convergence rates from the current variance including a complete random model: only 5 points are necessary to precisely describe the influence of random burying depth $d$ (RV3). Nearly zero levels of the current (mean and variance) below $0.03 \mu$ s involve instability of the convergence criterion (and positive SC gaps). Finally, Fig. 9 shows a good agreement between fully tensorized statistics of the current obtained with 343 points and the results given with 105 following previously depicted strategy.

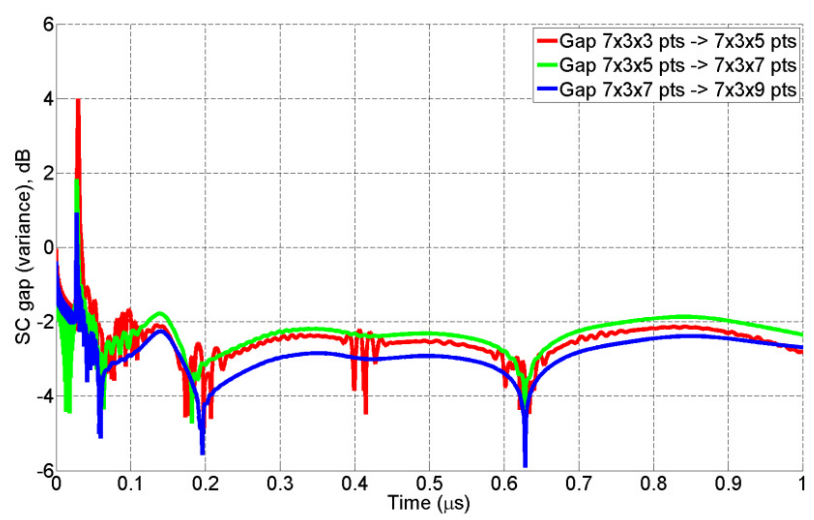

Figure 8: Relative gap with increasing SC orders (RV3).

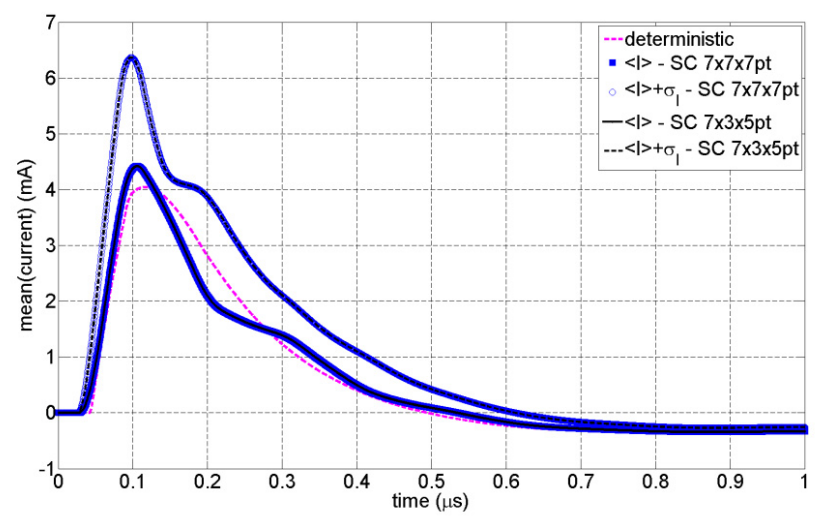

Figure 9: Current from fully tensorized SC ( $\left.7^{3} \mathrm{pts}\right)$ and asymmetrical number of points $(\sigma: 7, L: 3, d: 5)$. 


\section{Conclusion}

The coupling of deterministic time domain analytical solutions of the Pocklington integro-differential equation with stochastic collocation technique provides crucial information for the calculation of the response of wire configurations buried in lossy ground. The robustness, accuracy and convergence of the two techniques (deterministic and stochastic) ensure useful statistics for designing GPR systems by taking into account their intrinsic random characteristics (variations due to material parameters). Future work will be devoted to the analysis of benefit of the use of the space-time Pocklington equation coupled with proposed stochastic strategy in comparison with other costly sampling methods such as Monte Carlo.

\section{Acknowledgement}

This work benefited from networking activities carried out within the EU funded COST Action TU1208 “Civil Engineering Applications of Ground Penetrating Radar".

\section{References}

[1] L. Pajewski, Civil Engineering Applications of Ground Penetrating Radar, Rome: COST Action TU1208, 2013 - 2017.

[2] D. Poljak, Advanced Modeling in Computational Electromagnetic Compatibility, New Jersey: Wiley-Interscience, 2007.

[3] S. Lallechere, S. Sesnic, P. Bonnet, K. E. K. Drissi and D. Poljak, "Transient statistics from the lightning strike current flowing along grounding electrode," in 22nd International Conference on Software, Telecommunications and Computer Networks, Split, 2014.

[4] F. Paladian, P. Bonnet, S. Lallechere, A. Xemard and C. Miry, "On the numerical analysis of lightning effect on power installations," in International Colloquium on Lightning and Power Systems, Lyon, 2014.

[5] D. Poljak and N. Kovac, "Time domain modeling of a thin wire in a twomedia configuration featuring a simplified reflection/transmission coefficient approach," Engineering Analysis with Boundary Elements, vol. 33, pp. 283-293, 2009.

[6] T. Takashima, T. Nakae and R. Ishibashi, "Calculation of Complex Fields in Conducting Media," IEEE Transactions on Electrical Insulation, vol. 15, no. 1, pp. 1-7, February 1980.

[7] S. Šsnić, D. Poljak and S. Tkachenko, "Time domain analytical modeling of a straight thin wire buried in a lossy medium," Progress In Electromagnetics Research, vol. 121, pp. 485-504, 2011.

[8] D. Poljak, V. Doric, F. Rachidi, K. E. K. Drissi, K. Kerroum, S. V. Tkachenko and S. Sesnic, "Generalized Form of Telegrapher's Equations for the Electromagnetic Field Coupling to Buried Wires of Finite Length," 
IEEE Transactions on Electromagnetic Compatibility, vol. 51, no. 2, pp. 331-337, May 2009.

[9] D. Xiu, "Fast Numerical Methods for Stochastic Computations: A Review," Communications in Computational Physics, vol. 5, no. 2, pp. 242-272, 2009.

[10] H. Dodig, S. Lallechere, P. Bonnet, D. Poljak and K. El Khamlichi Drissi, "Stochastic sensitivity of the electromagnetic distributions inside a human eye modeled with a 3D hybrid BEM/FEM edge element method," Engineering Analysis with Boundary Elements, vol. 49, pp. 48-62, 2014.

[11] H. Bagci, A. C. Yucel, J. S. Hesthaven and E. Michielssen, "A Fast Stroud-Based Collocation Method for Statistically Characterizing EMI/EMC Phenomena on Complex Platforms," IEEE Transactions on Electromagnetic Compatibility, vol. 51, no. 2, pp. 301-311, 2009.

[12] P. M. Ivry, O. A. Oke, D. V. P. Thomas and M. Sumner, "Predicting Harmonic Distortion Level of a Voltage Source Converter at the PCC of a Grid," in 2nd International Symposium on Energy Challenges and Mechanics, Aberdeen, 2014. 